\title{
Teleophthalmology with optical coherence tomography imaging in community optometry. Evaluation of a quality improvement for macular patients
}

\author{
This article was published in the following Dove Press journal: \\ Clinical Ophthalmology \\ 30 November 2011 \\ Number of times this article has been viewed
}

\author{
Simon P Kelly' \\ lan Wallwork ${ }^{2}$ \\ David Haider ${ }^{\prime}$ \\ Kashif Qureshi' \\ 'Ophthalmology Department, Royal \\ Bolton Hospital National Health \\ Service Foundation Trust, Bolton, \\ ${ }^{2}$ Wallwork Opticians, Salford, UK
}

Correspondence: Simon P Kelly Ophthalmology Department, Royal Bolton Hospital National Health Service Foundation Trust, Bolton BL4 OJR, UK Tel +44 I204390694
Purpose: To describe a quality improvement for referral of National Health Service patients with macular disorders from a community optometry setting in an urban area.

Methods: Service evaluation of teleophthalmology consultation based on spectral domain optical coherence tomography images acquired by the community optometrist and transmitted to hospital eye services.

Results: Fifty patients with suspected macular conditions were managed via telemedicine consultation over 1 year. Responses were provided by hospital eye service-based ophthalmologists to the community optometrist or patient within the next day in 48 cases (96\%) and in $34(68 \%)$ patients on the same day. In the consensus opinion of the optometrist and ophthalmologist, 33 (66\%) patients required further "face-to-face" medical examination and were triaged on clinical urgency. Seventeen cases (34\%) were managed in the community and are a potential cost improvement. Specialty trainees were supervised in telemedicine consultations. Conclusion: Innovation and quality improvement were demonstrated in both optometry to ophthalmology referrals and in primary optometric care by use of telemedicine with spectral domain optical coherence tomography images. E-referral of spectral domain optical coherence tomography images assists triage of macular patients and swifter care of urgent cases. Teleophthalmology is also, in the authors' opinion, a tool to improve interdisciplinary professional working with community optometrists. Implications for progress are discussed.

Keywords: telemedicine, teleophthalmology, innovation, community referral, optical coherence tomography, service evaluation

\section{Introduction}

Telemedicine can be considered as the application of telecommunications technology to assist in delivering health services at a distance and is not new. ${ }^{1}$ Teleophthalmology was long considered as one of telemedicine's most challenging applications. ${ }^{2}$ Spectral domain ocular coherence tomography (SD OCT) imaging is being used in optometric primary care with referral of SD OCT images to hospital eye services (HES). Collaboration between primary and secondary care in the application of retinal imaging technology in this way, while innovative, is not unique. ${ }^{3,4}$ However no such previous publications included OCT imaging.

The primary care to specialist interface is a key organizational feature of many health care systems. Patients are referred to specialist care when investigations or therapeutic options are exhausted and more specialized care is needed, or thought to 
be needed. In United Kingdom ophthalmology practice, the community optometrist (CO) is usually the source of most referrals. Referral has considerable implications for patients, health care systems, and costs. There is evidence that referral processes can be improved. ${ }^{5,6}$ Currently, patients attending a CO in the authors' area, following general practitioner (GP) referral or self-referral, can expect waiting times of up to 2 days in the community. If urgency is indicated, a same day appointment is often offered. Those community optometric patients requiring forward referral by the GP for specialist ophthalmic opinion or treatment may, depending on the efficiency and work pressures at the GP's practice, perhaps wait several days for a referral to be made by the GP to an ophthalmologist. At present there is no reliable method for the $\mathrm{CO}$ to check that such referral has occurred or has been received by the HES. Critically there is a clinical risk of patient referrals being lost or delayed as a result of the multiple steps in referral. Furthermore, in the authors' experience, some GPs add little additional clinical information to the optometric referral. There is then often a possible additional waiting time of 18 weeks in the English National Health Service (NHS) for nonurgent cases. Further pathway delay can occur, even in urgent cases, as retinal patients are frequently first seen in the general clinic or "eye casualty" in the HES and only then often referred to a medical retina consultant. Several retinal conditions are time sensitive and require prompt treatment if good clinical outcomes are to be achieved. Timely access to NHS services for macular patients is under pressure and follow-up appointment capacity is problematic and is a patient safety concern. ${ }^{7}$ A recent study from Scotland highlighted further patient safety concern that optometrists find it difficult to accurately elicit the signs of macular disease. ${ }^{8}$

Traditional methods of retinal image transfer in the NHS are often unsatisfactory and described in Table 1. Digital transmission of clinical images is superior.

Optometrists undertake retinal examinations in the community and generally inform the patient's GP of any

Table I Transfer of photographed retinal images (traditional National Health Service methods)

- Faxing black and white images of colour clinical images to the recipient National Health Service hospital referral and booking management centre

- Sending black and white hard copy images (photocopies) or colour printouts from digital imaging equipment to the recipient using postal services, such as Royal Mail

- Burning clinical images onto compact disc for Royal Mail or courier delivery to the recipient detected eye disease. It is usually at the discretion of the GP (acting as a gatekeeper) to make a further referral (acting as a mailbox) to an ophthalmologist for further specialist review. Many GPs in the United Kingdom see merit in direct referral by COs as many GPs lack instruments for, or advanced training in, assessment of eye disorders. ${ }^{9,10}$

Many retinal conditions can be better diagnosed with SD OCT imaging. The objective in this report was to evaluate the novel service innovation of attaching SD OCT images to emailed clinical correspondence and consider implications for telemedicine ophthalmology consultation (teleophthalmology) involving patients with suspected macular disease. The authors were keen to improve triage of age-related macular degeneration (AMD) patients from optometry.

\section{Patients and methods}

This manuscript is an evaluation of 50 retinal e-consultations undertaken from June 2010 to August 2011 based on SD OCT imaging in the community. The setting was the Royal Bolton Hospital, Bolton and Wallwork Opticians, Salford. In contrast to many telemedicine reports involving rural and or remote areas, these locations are within 6 miles of each other, in Greater Manchester, United Kingdom. The patients referred were examined at the $\mathrm{CO}$ practice and all had, or were suspected of having, macular disorders. Patients attending for diabetic retinopathy screening were not included as separate local pathways exist for such patients.

The CO forwarded referrals with SD OCT images captured in primary care to an ophthalmologist for consideration, triage, advice, and diagnosis. A Topcon 3D OCT-2000 instrument (Topcon Corporation, Tokyo, Japan) was used by the CO. A color fundus image is included with OCT images captured from this instrument. OCT images were stored onsite at the optometrist's facility in the OCT instrument and offsite backup was made. The images were forwarded by secure email (NHSmail) and viewed by the ophthalmologist at the hospital or on a secure personal laptop computer. Images and text from the referrals were stored in a personal folder on the hospital NHS Foundation Trust's network in accordance with the Trust's Data Protection guidance. The $\mathrm{CO}$ also printed out the email correspondence and provided it on paper to the patients' GPs. OCT images were not provided to GPs. Trainee ophthalmologists were also able to participate in the e-consultation using secured smartphones. Clinical opinions from the trainees were reviewed by the consultant ophthalmologist.

The optometrist provided an explanation of the scheme to patients. Patient consent to transmission of their details 
was confirmed verbally by the optometrist. SD OCT imaging was conducted by the $\mathrm{CO}$ with the patient's permission and at an additional charge to the sight test fee. OCT imaging is not funded within the NHS General Ophthalmic Services sight test. If patients could not afford OCT imaging, the cost was born by the optometry practice. It is at the discretion of the $\mathrm{CO}$ to decide what further examinations are appropriate. ${ }^{11}$ An email assessment of the patients' consultation was sent by the $\mathrm{CO}$ if an ophthalmic opinion on suspected macular disease was required. Where appropriate, other clinical data such as previous color fundus images captured by the $\mathrm{CO}$ or descriptions of visual fields or Amsler testing were included. Each such e-referral was analyzed by the ophthalmologist. Where necessary, clarification was sought either by further email or telephone exchange. Patients who subsequently attended hospital had OCT imaging by nurses on the Zeiss Cirrus SD OCT (Carl Zeiss Meditec AG, Jena, Germany) instrument and other investigations as required.

\section{Results}

Telemedicine consultations based on SD OCT images were undertaken for 50 patients. The time taken to manage conditions was reduced compared to traditional methods. Specifically, in $96 \%$ of cases, analysis of the referrals and a working diagnosis/care pathway was provided by the ophthalmologist to the optometrist within the next calendar day (Tables 2 and 3).

The patient pathway is outlined in Figure 1. Where the teleophthalmology consultation recommended referral to the HES, the optometrist made recommendations to the GP for referral for further ophthalmic care. On occasion the telemedicine ophthalmologist advised direct urgent referral to HES based on the e-referral. This was either direct referral for "face-to-face" examination at the local HES in Bolton $(\mathrm{n}=30)$ or tertiary HES retinal care $(\mathrm{n}=2)$ in Manchester or by the GP $(n=1)$. If needed, the ophthalmologist contacted patients by telephone to arrange direct urgent attendance at the next day's ophthalmic clinic $(n=5)$. Seventeen cases (34\%) did not, in the consensus opinion of the optometrist and ophthalmologist, require medical review and were further

Table 2 Response period from receipt of optometric e-referral to reply by ophthalmologist

\begin{tabular}{lll}
\hline Response time & Number of referrals & Cumulative \\
\hline Same day & $34(68 \%)$ & $34(64 \%)$ \\
Next day & $14(28 \%)$ & $48(96 \%)$ \\
2 weeks & $2(4 \%)^{*}$ & $50(100 \%)$ \\
\hline
\end{tabular}

Note: *During holiday periods.
Table 3 Clinical detail of patients referred for teleophthalmology review

\begin{tabular}{lll}
\hline Diagnosis & Number & Percent \\
\hline Epiretinal membrane & 10 & 20 \\
Dry AMD & 9 & 18 \\
Vitreomacular traction & 8 & 16 \\
Wet AMD & 7 & 14 \\
Central serous chorioretinopathy & 3 & 6 \\
Choroidal nevi/melanoma & 3 & 6 \\
Macular schisis or hole & 3 & 6 \\
Other & 7 & 14 \\
TOTAL & 50 & 100 \\
\hline
\end{tabular}

Abbreviation: AMD, age-related macular degeneration.

managed in primary optometric care. In all cases the community SD OCT image quality was considered by the ophthalmologist to be as good as, or better than, the SD OCT images captured by the hospital nursing staff. In two cases the community SD OCT image was superior to that captured in hospital clinic on initial attempt. Figure 2 depicts an example of a wet AMD case imaged at the CO's practice and at the hospital clinic on successive days. Patient satisfaction was not formally accessed, but it was noted that most patients spontaneously remarked on how satisfied they were.

e-referral pathway

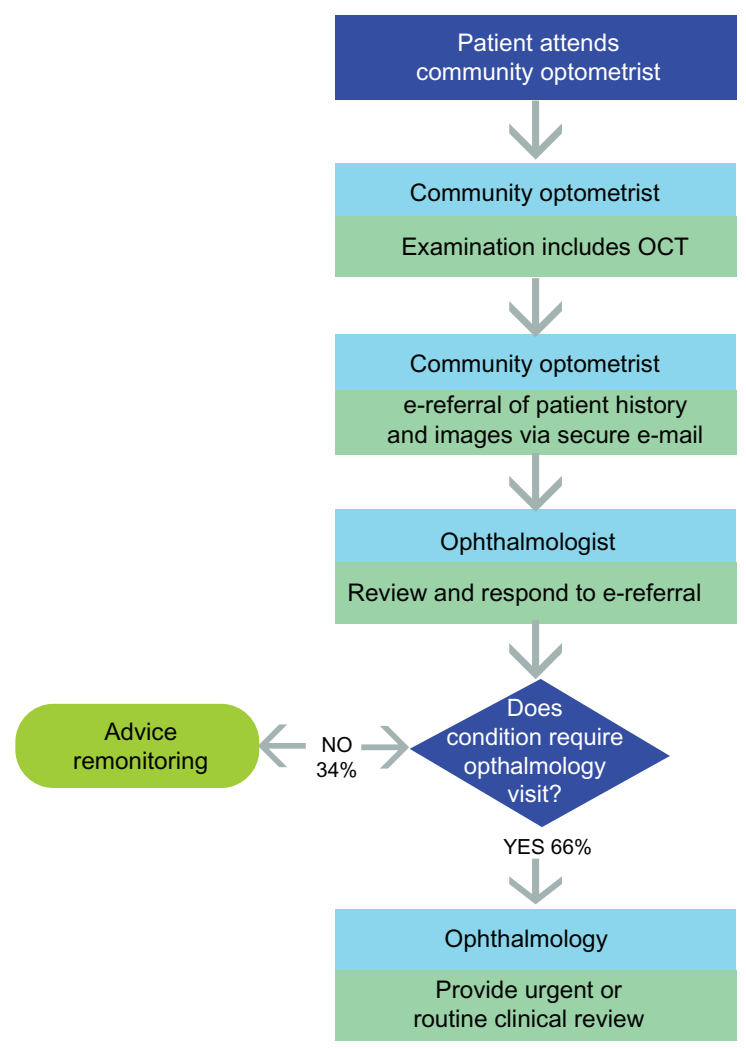

Figure I Outline of teleophthalmology patient pathway. Abbreviation: OCT, optical coherence tomography. 


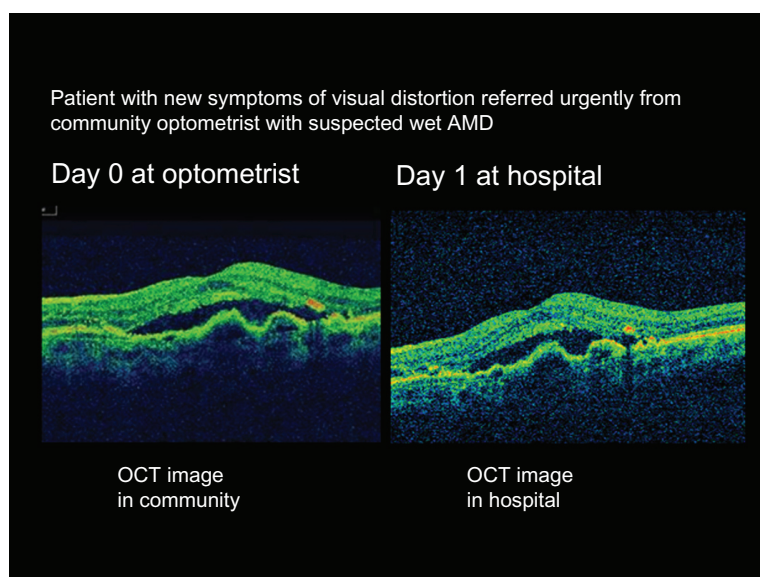

Figure 2 Optical coherence tomography (OCT) fundus image of wet age-related macular degeneration (AMD) patient taken on OCT instrument in community optometry care (left panel) and again in hospital care (right panel) on the following day.

Many patients expressed surprise that telemedicine of OCT images is not widespread.

\section{Discussion}

E-health and telemedicine has the potential for significant improvement in the quality and productivity of patient care compared to traditional methods. Randomized controlled trial evaluation of their effect would be ideal but are rare. Service evaluation is conducted to judge current care and thus differs from clinical research or clinical audit inter alia in that no randomization is undertaken. ${ }^{12} \mathrm{~A}$ recent service evaluation showed e-consultation with hospital nephrologists promotes effective management of patients with mild-to-moderate chronic kidney disease in primary care. ${ }^{13}$ Similarly in the current service evaluation retinal referrals from optometry to hospital ophthalmologists were reduced by e-consultation. This reduction allowed swifter attention to referred cases and, importantly, urgent attention to clinically urgent retinal cases. Benefits also included more efficient triage and prioritization of referrals and appointments at the hospital. The benefits in the community included ease of access to OCT scanning at one-stop visits, enhanced education of optometrists in the care of retinal patients, and improved interdisciplinary professional working. This innovation has the potential to support the Quality, Innovation, Productivity and Prevention scheme. ${ }^{14}$ With respect to suspected wet AMD patients, direct faxed referral from the community to the HES is endorsed by relevant stakeholders. ${ }^{15}$ Email is faster and superior in quality to such faxed and often handwritten optometric or GP urgent referral documents. Specifically, typed text is clearer than handwritten text and the author and date of writing are easy to determine. In the authors' opinion, this quality and speed improvement enables better prioritization. Secondly, it is also often at the discretion of the GP and or ophthalmologist to inform the referring optometrist with the outcome if referred to HES. Importantly, despite written patient consent, such feedback to COs is often lacking. ${ }^{16}$ Such disconnect has the potential to lead to breakdown in continuity of care at the patient's next visit to the $\mathrm{CO}$ and may lead to further referrals. Poor communication may also represent low productivity or "waste" in the healthcare system and a lost opportunity for multidisciplinary education and audit. Furthermore as well as providing savings to the NHS by reduced hospital and GP clinic visits, this service development also benefits the economy and ecology by reduced patient travel and importantly reduces patient concern by swift response to consultation.

It is not intended that teleophthalmology would replace "face-to-face" consultation; rather, electronic referral of OCT images is, in the authors' opinion, a useful tool to assist, prioritize, and refine referral of retinal patients in the digital age. The proportion of referrals from optometrists compared to those from GPs is increasing. ${ }^{17}$ Currently, there are fewer ophthalmologists in the United Kingdom, pro rata, than in any other European Union nation. Together with a high level of undetected, yet treatable visual morbidity and an increasing elderly and diabetic population, improvements in primary eye care are also needed. Treatment of wet AMD patients requires regular intravitreal injections and is an area of high volume care under pressure in England and Wales. ${ }^{7}$ In the authors' opinion, teleophthalmology may assist with such pressures.

A limitation of this service review is that it was restricted to macular patients. A recent report described a pilot teleconsultation network of general and specialist ophthalmologists in Italy for retinal cases. ${ }^{18}$ Others have used teleophthalmology in glaucoma, acute eye conditions, retinopathy of prematurity, and strabismus. ${ }^{19-22}$ There is merit of teleophthalmology in challenging locations, such as in rural areas, ${ }^{3,20}$ prisons, ${ }^{23}$ and in military settings. ${ }^{24}$

This project did not include diabetic patients, as separate pathways for such patients exist locally within diabetic retinal screening services. The potential for teleophthalmology with OCT in such diabetic retinal screening services, and which are now embedded in the NHS, requires further analysis.

The present evaluation of a pilot scheme highlights the potential wider application of innovation in clinical imaging and information technology transfer such as has occurred within picture archiving and communication systems. Access to NHSmail by optometrists is available from the NHS on request and this requires publicity and adoption within the 
optometry profession. Optometry connection to the NHS N3 network - for picture archiving and communication systems transfer - and or use of image exchange portals are further quality improvements that could be rolled out. If teleophthalmology services do develop, it is important that they are compliant with data protection and patient safety aspirations. Teleradiology is perhaps the most developed area of telemedicine. The comprehensive guidelines on teleradiology services developed by the Royal College of Radiologists may be of merit to those seeking to roll out teleophthalmology services. ${ }^{25}$ Teleophthalmology consultation with community optometry enables ophthalmologists to focus on macular patients with significant/urgent disease and, if applied more widely, could reduce referrals, costs, and waiting times for such services in the United Kingdom. A limitation is that a $24 / 7 / 365$ rapid response teleophthalmology service is unlikely to be sustained unless motivation or remuneration is provided to those ophthalmologists, optometrists, and providers involved. This innovation would therefore need to be recognized in service planning or commissioning. In conclusion, this service evaluation indicates the value of ophthalmic images transfer between primary and secondary care in the better management of patients with macular conditions. Benefits to both patient care and interprofessional working in an urban area have occurred.

\section{Conclusion}

\section{What was known before}

- Telemedicine is of known benefit in image dependent specialties such as radiology and ophthalmology.

- In England, remote review of ocular fundus images is in use to underpin NHS diabetic retinopathy screening services.

- Delays and service pressures are causing problems for retinal patient care in the NHS.

\section{What this study adds}

- The demonstration of quality improvement in NHS optometry/ophthalmology referral by use of telemedicine.

- The value of OCT telemedicine consultation in primary care for retinal disease.

- The merits of teamwork in community optometric and hospital opthalmic practice for patients using innovative technology.

\section{Acknowledgment}

We are grateful to John Sparrow, Susan Blakeney, Cindy Tromans, and Amir Hannan for comments on a draft of this service evaluation.

\section{Contributors}

I Wallwork undertook the clinical data collection and imaging in primary care. All authors were involved in data analysis and jointly wrote the paper. SP Kelly conceived the study idea and is the guarantor.

\section{Disclosure}

The authors report no conflicts of interest in this work.

\section{References}

1. Higgins C, Dunn E, Conrath D. Telemedicine: an historical perspective. Telecomm Policy. 1984;8:307-313.

2. Li HK. Telemedicine and Ophthalmology. Surv Ophthalmol. 1999; 44(1):61-72.

3. Cameron JR, Ahmed S, Curry P, Forrest G, Sanders R. Impact of direct electronic optometric referral with ocular imaging to a hospital eye service. Eye (Lond). 2009;23(5):1134-1140.

4. Hanson C, Tennant MT, Rudnisky CJ. Optometric referrals to retina specialists: evaluation and triage via teleophthalmology. Telemed $J E$ Health. 2008;14(5):441-445.

5. Akbari A, Mayhew A, Al-Alawi MA, et al. Interventions to improve outpatient referrals from primary care to secondary care. Cochrane Database System Rev. 2008;4:CD005471.

6. Jones NP, Lloyd IC, Kwartz J. General practitioner referrals to an eye hospital: a standard referral form. J R Soc Med. 1990;83(12): 770-772.

7. Kelly SP, Barua A. A review of safety incidents in England and Wales for vascular endothelial growth factor inhibitor medications. Eye (Lond). 2011;25(6):710-716.

8. Muen WJ, Hewick SA. Quality of optometry referrals to neovascular age-related macular degeneration clinic: a prospective study. JRSM Short Rep. 2011;2(8):64.

9. Shuttleworth GN, Marsh GW. How effective is undergraduate and postgraduate teaching in ophthalmology? Eye (Lond). 1997;11(Pt 5): 744-750.

10. Featherstone PI, James C, Hall MS, Williams A. General practitioners' confidence in diagnosing and managing eye conditions: a survey in south Devon. Br J Gen Pract. 1992;42(354):21-24.

11. General Optical Council. The rules relating to injury or disease of the eye. 1999. Available from: https://www.optical.org/goc/filemanager/root/ site_assets/legislation/rules_and_regulations/injurydisease 1999.pdf. Accessed October 31, 2011.

12. National Research Ethics Service, National Patient Safety Agency. Defining research. November 29, 2007. Available from: http://www. nres.npsa.nhs.uk/EasySiteWeb/GatewayLink.aspx?alId=355. Accessed October 31, 2011.

13. Stoves J, Connolly J, Cheung CK, et al. Electronic consultation as an alternative to hospital referral for patients with chronic kidney disease: a novel application for networked electronic health records to improve the accessibility and efficiency of healthcare. Qual Saf Health Care. 2010;19(5):e54.

14. Department of Health. Quality, innovation, productivity and prevention (QIPP). June 23, 2011. Available from: http://www.dh.gov.uk/en/Healthcare/ Qualityandproductivity/QIPP/index.htm. Accessed October 31, 2011.

15. Royal College of Ophthalmologists. Commissioning contemporary AMD services: a guide for commissioners and clinicians. July 2007. Available from: www.rcophth.ac.uk/core/core_picker/download. asp?id=181. Accessed October 31, 2011.

16. Evans BJ, Harle DE, Cocco B. Optometric referrals: towards a two way flow of information? Br J Ophthalmol. 2005;89(12):1663.

17. Davey CJ, Green C, Elliott DB. Assessment of referrals to the hospital eye service by optometrists and GPs in Bradford and Airedale. Ophthalmic Physiol Opt. 2011;31(1):23-28. 
18. Azzolini C. A pilot teleconsultation network for retinal diseases in ophthalmology. J Telemed Telecare. 2011;17(1):20-24.

19. Tuulonen A, Ohinmaa T, Alanko HI, Hyytinen P, Juutinen A, Toppinen E. The application of teleophthalmology in examining patients with glaucoma: a pilot study. J Glaucoma. 1999;8(6):367-373.

20. Kulshrestha MK, Lewis D, Williams C, Axford A. A pilot trial of teleophthalmology services in north Wales. JTelemed Telecare. 2010;16(4): 196-197.

21. Richter GM, Williams SL, Starren J, Flynn JT, Chiang MF. Telemedicine for retinopathy of prematurity diagnosis: evaluation and challenges. Surv Ophthalmol. 2009;54(6):671-685.

22. Cheung JC, Dick PT, Kraft SP, Yamada J, Macarthur C. Strabismus examination by telemedicine. Ophthalmology. 2000;107(11): 1999-2005.
23. Barry CJ, Henderson C, Kanagasingam Y, Constable IJ. Working toward a portable tele-ophthalmic system for use in maximum-security prisons: a pilot study. Telemed J E Health. 2001;7(3):261-265.

24. Mines MJ, Bower KS, Lappan CM, Mazzoli RA, Poropatich RK. The United States Army Ocular Teleconsultation Program 2004 through 2009. Am J Ophthalmol. 2011;152(1):126-132.

25. Royal College of Radiologists. Standards for the provision of teleradiology within the United Kingdom. May 2010. Available from: http:// www.rcr.ac.uk/docs/radiology/pdf/BFCR(10)7_Stand_telerad.pdf. Accessed October 31, 2011.
Clinical Ophthalmology

\section{Publish your work in this journal}

Clinical Ophthalmology is an international, peer-reviewed journal covering all subspecialties within ophthalmology. Key topics include: Optometry; Visual science; Pharmacology and drug therapy in eye diseases; Basic Sciences; Primary and Secondary eye care; Patient Safety and Quality of Care Improvements. This journal is indexed on

\section{Dovepress}

PubMed Central and CAS, and is the official journal of The Society of Clinical Ophthalmology (SCO). The manuscript management system is completely online and includes a very quick and fair peer-review system, which is all easy to use. Visit http://www.dovepress.com/ testimonials.php to read real quotes from published authors. 